\title{
Association of Differing Qatari Genotypes with Vitamin D Metabolites
}

\author{
Youssra Dakroury, ${ }^{1}$ Alexandra E. Butler ${ }^{D},{ }^{2}$ Soha . Dargham, ${ }^{1}$ Aishah Latif, ${ }^{3}$ Amal Robay, ${ }^{1}$ \\ Ronald G. Crystal, ${ }^{4}$ and Stephen L. Atkin ${ }^{1,5}$ \\ ${ }^{1}$ Weill Cornell Medicine-Qatar, P.O. Box 24144, Doha, Qatar \\ ${ }^{2}$ Diabetes Research Center (DRC), Qatar Biomedical Research Institute (QBRI), Hamad Bin Khalifa University (HBKU), \\ Qatar Foundation (QF), Doha, Qatar \\ ${ }^{3}$ Antidoping Laboratory, Doha, Qatar \\ ${ }^{4}$ Department of Genetic Medicine, Weill Cornell Medicine, New York, USA \\ ${ }^{5}$ Royal College of Surgeons Ireland, Busaiteen, Bahrain
}

Correspondence should be addressed to Alexandra E. Butler; aeb91011@gmail.com

Received 30 December 2019; Revised 5 March 2020; Accepted 13 March 2020; Published 13 April 2020

Academic Editor: Flavia Prodam

Copyright (c) 2020 Youssra Dakroury et al. This is an open access article distributed under the Creative Commons Attribution License, which permits unrestricted use, distribution, and reproduction in any medium, provided the original work is properly cited.

\begin{abstract}
Objective. Genetic studies have identified four Qatari genotypes: Q1 Arab, Bedouin; Q2 Asian/Persian; Q3 African; and a fourth admixed group not fitting into the previous 3 groups. This study was undertaken to determine if there was an increased risk of deficiency of vitamin D and its metabolites associated with differing genotypes, perhaps due to genetic differences in skin pigmentation. Methods. 398 Qatari subjects (220 type 2 diabetes and 178 controls) had their genotype determined by Affymetrix $500 \mathrm{k}$ SNP arrays. Total values of 1,25-dihydroxyvitamin D (1,25(OH)2D), 25-hydroxyvitamin D (25(OH)D), 24,25-dihydroxyvitamin D (24,25(OH)2D), and 25-hydroxy-3epi-vitamin D (3epi-25(OH)D) concentrations were measured by the LC-MS/ MS analysis. Results. The distribution was as follows: 164 (41.2\%) genotyped Q1, 149 (37.4\%) genotyped Q2, 31 (7.8\%) genotyped Q3, and 54 (13.6\%) genotyped "admixed." Median levels of 25(OH)D and 3epi-25(OH)D did not differ across Q1, Q2, Q3, and "admixed" genotypes, respectively. 1,25(OH)2D levels were lower $(p<0.04)$ between Q2 and the admixed groups, and 24,25(OH) $2 \mathrm{D}$ levels were lower $(p<0.05)$ between Q1 and the admixed groups. Vitamin D metabolite levels were lower in females for $25(\mathrm{OH}) \mathrm{D}, 1,25(\mathrm{OH}) 2 \mathrm{D}(p<0.001)$, and $24,25(\mathrm{OH}) 2 \mathrm{D}(p<0.006)$, but 3epi-25(OH)D did not differ $(p<0.26)$. Diabetes prevalence was not different between genotypes. Total 1,25(OH)2D $(p<0.001)$, total $24,25(\mathrm{OH}) 2 \mathrm{D}(p<0.001)$, and total 3epi$25(\mathrm{OH}) \mathrm{D}(p<0.005)$ were all significantly lower in diabetes patients compared to controls whilst the total 25(OH)D was higher in diabetes than controls $(p<0.001)$. Conclusion. Whilst 25(OH)D levels did not differ between genotype groups, 1,25(OH)2D and $24,25(\mathrm{OH}) 2 \mathrm{D}$ were lower in the admixed group, suggesting that there are genetic differences in vitamin $\mathrm{D}$ metabolism that may be of importance in a population that may allow a more targeted approach to vitamin $\mathrm{D}$ replacement. This may be of specific importance in vitamin D replacement strategies with the Q2 genotype requiring less, and the other genotypes requiring more to increase $1,25(\mathrm{OH}) 2 \mathrm{D}$. Whilst overall the group was vitamin $\mathrm{D}$ deficient, total $25(\mathrm{OH}) \mathrm{D}$ was higher in diabetes, but $1,25(\mathrm{OH}) 2 \mathrm{D}$, $24,25(\mathrm{OH}) 2 \mathrm{D}$, and 3epi-25(OH)D were lower in diabetes that did not affect the relationship to genotype.
\end{abstract}

\section{Introduction}

Next generation exome sequencing has identified three major genetic subgroups within the Qatari population (Q1 Bedouin, Q2 Persian-South Asian, and Q3 African) and has identified variants within genes that have effects on clinically significant Mendelian diseases [1-3]. Several of these Mendelian variants were only segregating in one Qatari subpopulation [2]. However, because of their initial origin, this may lead to differences in skin pigmentation that would potentially lead to differences in vitamin $\mathrm{D}$ akin to those with darker skins becoming vitamin $\mathrm{D}$ deficient in the northern 
hemisphere [4]. This, in turn, may exacerbate the already prevalent vitamin D deficiency seen in the Middle East and, in particular, in Qatar.

Vitamin D deficiency has become the most common nutritional deficiency throughout the world [5]. While vitamin D deficiency is a global issue [6], cultural norms dictating full body coverage in parts of the world such as the Middle East magnify the issue of vitamin D deficiency in these regions [7, 8]. Vitamin D is key in maintaining skeletal health, but it has been found to have a wider role in, for example, supporting cardiovascular health, and it may exert anticancer effects [9]. Tissue specific production of $1,25(\mathrm{OH})_{2} \mathrm{D}$ from its precursor $25(\mathrm{OH}) \mathrm{D}$ is essential for proper functioning of both the adaptive and the innate immune systems [10]. Vitamin D sufficiency is important for cellular and tissue homeostasis, and it has been shown to be associated with reduced all-cause mortality [11]. Obesity, which is a particular issue in the Middle East, can lead to decreased bioavailability of vitamin $\mathrm{D}$ and, therefore, deficiency because of deposition in the body fat compartments [12].

Vitamin D3 (cholecalciferol) is endogenously produced whilst vitamin D2 is derived from the diet as ergosterol, primarily from mushrooms and fungi, which is then converted to ergocalciferol by UV-B; both are hydroxylated to vitamin $\mathrm{D} 2\left(25(\mathrm{OH}) \mathrm{D}_{2}\right)$ or vitamin $\mathrm{D} 3\left(25(\mathrm{OH}) \mathrm{D}_{3}\right)$ by multiple 25-hydroxylases $[13,14]$ in the liver. Vitamin D is transported to the kidney and converted either to the active $1,25(\mathrm{OH})_{2} \mathrm{D}$ by 1 alpha hydroxylase or to $24,25(\mathrm{OH})_{2} \mathrm{D}$ [15].

It has been recently reported that extrarenal tissues may also convert $25(\mathrm{OH}) \mathrm{D}$ to $1,25(\mathrm{OH})_{2} \mathrm{D}$ [16]. $1,25(\mathrm{OH})_{2} \mathrm{D}$ binds to the vitamin $\mathrm{D}$ receptor (VDR) and subsequently heterodimerizes with the retinoid $\mathrm{X}$ receptor for its action [15]. Vitamin D2 is available both as a supplement and as a pharmaceutical to treat vitamin D deficiency.

3 epi-25(OH)D is formed by 3 -epimerase $[13,17]$ and is relatively inactive compared with $25(\mathrm{OH}) \mathrm{D}$, the main issue being that it may be measured inadvertently whilst measuring $25(\mathrm{OH}) \mathrm{D}$, leading to $25(\mathrm{OH}) \mathrm{D}$ overestimation [18]. However, the 3 -epimer may be as potent as $1,25(\mathrm{OH})_{2} \mathrm{D}_{3}$ on PTH suppression $[13,19]$, but there is less data on any other biological effects it may possess.

Epidemiological evidence has related vitamin D deficiency to an increased risk of type 2 diabetes (T2DM) $[20,21]$, with vitamin $\mathrm{D}$ deficiency associated with both insulin resistance and beta cell dysfunction [22], contributing to metabolic syndrome and T2DM [23, 24]. Vitamin D has been associated with poorer glycemic control and increased mortality [25], and given the marked vitamin D deficiency in this population [26], this may lead to increased microvascular complications of retinopathy, neuropathy, and nephropathy $[27,28]$.

We hypothesized that the skin pigmentation associated with the differing Qatari genotypes would have an impact on vitamin $\mathrm{D}$ and its metabolites, perhaps exacerbated by the presence of diabetes, which may have major implications on vitamin D replacement strategies, and therefore, this study was undertaken.

\section{Methods}

2.1. Study Population. The recruitment strategy for the subjects in this study has been described before [3]. Briefly, all subjects were over the age of 30 , and there were a minimum of three generations Qatari. Cases were excluded if any of the following were present: history of type 1 diabetes, maturity onset diabetes of the young (MODY), maternally inherited diabetes and deafness syndrome (MIDD), a first degree relative with type 1 diabetes, or secondary diabetes [3]. The diagnosis of type 2 diabetes was made according to the WHO guidelines [29]; for inclusion in the type 2 diabetes cohort, at least one of the following was required: fasting plasma glucose $>7 \mathrm{mmol} / \mathrm{l}, \mathrm{HbAlc}>6.5 \%$, or a diagnostic glucose tolerance test. Inclusion in the nondiabetic control group required a normal glucose tolerance test. 398 subjects with no known familial relationships and satisfying the Qatari ancestry criteria and with unambiguous assignment to a Qatari subpopulation were selected from a group visiting the health clinics at Hamad Hospital, Doha, Qatar, for a routine diabetes screening [2]. Nondiabetic subjects were comprised of relatives accompanying the type 2 diabetic (T2DM) subjects. A total of 398 subjects were genotyped (Table 1).

Diabetes patients were on hypoglycemic therapy, and all subjects had been prescribed vitamin $\mathrm{D}_{2}$ supplements 50,000 units weekly.

The study was approved by Weill Cornell IRB (IRB\# 1300063), and all participants provided written informed consent. The conduct of the trial was in accordance with ICH GCP and the Declaration of Helsinki.

2.2. Study Design. Following an overnight fast, blood samples were collected, and weight and blood pressure were measured at the baseline visit. The fasting venous blood was collected into fluoride oxalate and serum gel tubes. Samples were separated by centrifugation at $2000 \times \mathrm{g}$ for 15 minutes at $4^{\circ} \mathrm{C}$, and the aliquots were stored at $-80^{\circ} \mathrm{C}$ within 1 hour of collection. Overnight urine samples were collected, and aliquots were stored at $-80^{\circ} \mathrm{C}$ until batch analysis. Blood pressure was measured using an automated device (NPB-3900; Nellcor Puritan Bennett, Pleasanton, CA) during each study visit. Blood pressure measurements were performed after the subjects had been seated quietly for at least 5 minutes and with the right arm supported at heart level. Three readings were taken, each at least 2 minutes apart, and then the average of the readings was obtained.

2.3. Serum Vitamin D Measurement. Serum vitamin D levels were quantified using isotope-dilution liquid chromatography tandem mass spectrometry (LC-MS/MS). $25 \mu \mathrm{L}$ of internal standards (d6-1calcitriol $(1.5 \mathrm{ng} / \mathrm{mL}), \mathrm{d} 6-25 \mathrm{OHD}_{3}$ $(50 \mathrm{ng} / \mathrm{mL})$, and $\mathrm{d} 6-25 \mathrm{OHD}_{2}(20 \mathrm{ng} / \mathrm{mL})$ were added into each microcentrifuge tube containing $250 \mu \mathrm{L}$ of calibration standards, quality control, or serum samples and kept for 30 minutes to reach binding equilibrium. The samples were diluted with $250 \mu \mathrm{L}$ of pretreatment solution (isopropanol 
TABle 1: Demographics of the 398 subjects.

\begin{tabular}{lc}
\hline & Population \\
\hline Age, mean (SD) & $49.8(10.6)$ \\
Gender, $N(\%)$ & \\
$\quad$ Male & $226(56.8)$ \\
$\quad$ Female & $172(43.2)$ \\
HBA1C, median (IQR) & $6.9(2.7)$ \\
Glucose, median (IQR) & $6.1(3.9)$ \\
Diabetes-yes, $N(\%)$ & $220(55.3)$ \\
Hypertension-yes, $N(\%)$ & $186(46.7)$ \\
Dyslipidemia-yes, $N(\%)$ & $224(56.3)$ \\
\hline
\end{tabular}

and water; $50: 50 \mathrm{v} / \mathrm{v}$ ) and left to stand for at least $15 \mathrm{~min}$ to displace binding protein.

$300 \mu \mathrm{L}$ of pretreated samples were loaded onto ISOLUTE $^{\circledR}$ supported liquid extraction (SLE+) columns (Biotage), followed by elution with $1.8 \mathrm{~mL}$ of $\mathrm{n}$-heptane $(2 \times 900 \mu \mathrm{L})$ into a collection tube already containing $200 \mu \mathrm{L}$ of $0.25 \mathrm{mg} / \mathrm{mL}$ PTAD solution in ethyl acetate and heptane $(8: 92 \mathrm{v} / \mathrm{v})$. The eluate was evaporated to dryness using turbovap under nitrogen gas heated at $38^{\circ} \mathrm{C}$. Once dried, 50 $\mu \mathrm{L}$ of reconstituted solution consisting of methanol and deionized water, $70: 30 \mathrm{v} / \mathrm{v}$, and $0.006 \%$ methylamine were added into all tubes. The derivatised extracts were transferred into LC insert vials and $10 \mu \mathrm{L}$ from each was injected into the LC-MS/MS system. Vitamin D deficiency was defined according to the Endocrine Society: deficiency, insufficiency, and repletion as $\leq 20 \mathrm{ng} / \mathrm{mL}, 20-30 \mathrm{ng} / \mathrm{mL}$, and $\geq 30 \mathrm{ng} / \mathrm{mL}$, respectively.

2.4. Qatari Genetic Subpopulation Genotyping. DNA was extracted from blood using the QIAamp DNA Blood Maxi Kit (Qiagen Sciences Inc, Germantown, MD). The 398 subjects were classified into the three genetic subgroups described in the Qatari population $[8,9]$ using a TaqMan SNP Genotyping Assay (Life Technologies, Carlsbad, CA) for a previously described panel of 48 ancestry informative SNPs $[8,9]$. Average genotype call rate was $96 \%$ and analyzed in STRUCTURE with $K=3$. Q1, Q2, or Q3 population was assigned if the highest proportion was $>65 \%$; otherwise, they were classed as "admixed".

\subsection{Study Outcomes}

2.5.1. Statistical Analyses. Data trends were visually and statistically evaluated for normality. Nonparametric tests (Mann-Whitney U and Kruskall-Wallis tests) were applied on data that violated the assumptions of normality when tested using the Kolmogorov-Smirnov test. Bonferroni correction was applied to account for multiple testing. Statistical analysis was performed using SPSS for Windows, version 24.0. All values are given as mean \pm SD or as mean with $95 \%$ confidence interval (CI) unless otherwise specified.

\section{Results}

3.1. Baseline Characteristics. The baseline characteristics for the entire cohort and those genotyped are shown in Table 1.
It can be seen that there were no differences in age, gender, or BMI between the differing genotypes; however, the Q3 genotype had significantly more hypertension in the diabetes population $(p<0.04)$. Vitamin D status between males and females is shown in supplementary Table 1: $25(\mathrm{OH}) \mathrm{D}$, $1,25(\mathrm{OH}) 2 \mathrm{D}(p<0.001)$ and $24,25(\mathrm{OH}) 2 \mathrm{D}(p<0.006)$ were significantly lower in females compared to males, but 3epi$25(\mathrm{OH}) \mathrm{D}$ did not differ $(p<0.26)$.

3.2. Vitamin D in relation to Qatari Genotype. The relationship of the Qatari genotypes to diabetes and vitamin D metabolites are shown in Table 2. Vitamin D metabolites between the diabetes and control subjects are shown in Table 3.

In the 398 subjects, the mean age was 49.8 years and $56.8 \%$ were males (Table 1 ). The distribution was as follows: $164(41.2 \%)$ genotype Q1, $149(37.4 \%)$ genotype Q2, 31 (7.8\%) genotype Q3, and 54 (13.6\%) genotyped "admixed". Median levels of $25(\mathrm{OH}) \mathrm{D}, 1,25(\mathrm{OH})_{2} \mathrm{D}$, and 3epi-25(OH) D did not differ across Q1, Q2, Q3, and "admixed" genotypes, respectively. $1,25(\mathrm{OH})_{2} \mathrm{D}$ levels significantly differed between Q2 and the admixed groups $(p<0.04)$, and $24,25(\mathrm{OH})_{2} \mathrm{D}$ significantly differed between $\mathrm{Q} 1$ and the admixed groups $(p<0.05)$ (Table 2$)$.

Vitamin D metabolites and the relation to genotype was determined for the entire group that included subjects with and without diabetes, but as there was no difference in the association of diabetes with genotype, then this was a valid approach. The vitamin $\mathrm{D}$ levels in control and diabetes subjects are shown in Table 3. Overall the control group was vitamin D deficient (in accord with the Endocrine Society definition) with the median total $25(\mathrm{OH}) \mathrm{D}$ being $19.58 \mathrm{ng} / \mathrm{ml}$ (59.32) and the diabetes group vitamin $\mathrm{D}$ insufficient $26.46 \mathrm{ng} / \mathrm{ml}(17.84)$ : total $25(\mathrm{OH}) \mathrm{D}$ was higher in diabetes $\quad(p<0.001)$, but $1,25(\mathrm{OH})_{2} \mathrm{D} \quad(p<0.001)$, $24,25(\mathrm{OH})_{2} \mathrm{D}(p<0.001)$, and 3epi-25(OH)D $(p=0.005)$ were lower in diabetes (Table 3 ).

\section{Discussion}

This study clearly showed that the differing Qatari genotypes did not contribute to the levels of measurable $25(\mathrm{OH}) \mathrm{D}$ and each was as equally vitamin D deficient as the other. On one hand, this is surprising given the differing degrees of skin pigmentation [4]. Studies on Qatari genotype revealed 3 genotypes: Q1 Arab, Bedouin; Q2 Asian/Persian; and Q3 African, with Q3 likely having the most pigmented skin [1]. However, given the whole body coverage that is prevalent in the Middle East [7], then that is likely to be the prevailing and dominant factor in this instance rather than skin pigmentation. Vitamin D in the form of $25(\mathrm{OH}) \mathrm{D}$ is measured to ascertain vitamin $\mathrm{D}$ sufficiency [30] with the other metabolites being largely measured for research purposes [30]. In this case, $1,25(\mathrm{OH}) 2 \mathrm{D}$ levels significantly differed between Q2 and the admixed groups, perhaps through increased renal or extrarenal CYP27B1 activity, whilst $24,25(\mathrm{OH}) 2 \mathrm{D}$ significantly differed between Q1 and the admixed groups, perhaps through modulation of CYP27A1 
A strength of the study is the measurement of the vitamin D metabolites by state of the art methods. It is well recognized that lifestyle and diet are important for vitamin $\mathrm{D}$ levels, and whilst all of the subjects were more than 3 generation Qataris who all followed whole body coverage and the Qatari lifestyle, a limitation of the study was that formal dietary and lifestyle assessment was not undertaken. Other limitations include the cross sectional nature of the study, and it is unknown if the vitamin $\mathrm{D}$ range for each metabolite was the same in each genotype though the interquartile ranges for each were no different for each metabolite. An additional limitation was that the estimated glomerular filtration rate was not measured that could have impacted on vitamin $\mathrm{D}$ metabolite levels.

In conclusion, 25(OH)D levels did not differ between groups, suggesting that skin coloration may not contribute to vitamin $\mathrm{D}$ status; however, the $1,25(\mathrm{OH})_{2} \mathrm{D}$ form was higher for Q2 and 24,25(OH $)_{2} \mathrm{D}$ was higher in Q1 compared to the "admixed" group, suggesting that there are genetic differences in vitamin D metabolism that may be of importance in this population particularly if increased supplementation is needed for differing genotypes.

\section{Data Availability}

All data relating to this study will be made available by the corresponding author upon reasonable request.

\section{Conflicts of Interest}

The authors declare that they have no conflicts of interest.

\section{Authors' Contributions}

Ronald G. Crystal, Stephen L. Atkin contributed equally to this work. YD and AEB researched the data and wrote the manuscript. SRD performed the statistical analysis. AL performed the vitamin $\mathrm{D}$ measurements. AR researched data. RGC and SLA designed the study and contributed to the discussion.

\section{Acknowledgments}

The publication of this article was funded by the Qatar National Library.

\section{Supplementary Materials}

Supplementary Table 1: vitamin D metabolites according to gender. (Supplementary Materials)

\section{References}

[1] H. Hunter-Zinck, S. Musharoff, J. Salit et al., "Population genetic structure of the people of Qatar," The American Journal of Human Genetics, vol. 87, no. 1, pp. 17-25, 2010.

[2] J. L. Rodriguez-Flores, K. Fakhro, N. R. Hackett et al., "Exome sequencing identifies potential risk variants for Mendelian disorders at high prevalence in Qatar," Human Mutation, vol. 35, no. 1, pp. 105-116, 2014.
[3] S. L. O’Beirne, J. Salit, J. L. Rodriguez-Flores et al., "Type 2 diabetes risk allele loci in the Qatari population," PloS one, vol. 11, no. 11, Article ID e0156834, 2016.

[4] A. R. Webb, A. Kazantzidis, R. C. Kift, M. D. Farrar, J. Wilkinson, and L. E. Rhodes, "Colour counts: sunlight and skin type as drivers of vitamin D deficiency at UK latitudes," Nutrients, vol. 10, no. 4, 2018.

[5] B. Rudick, S. Ingles, K. Chung, F. Stanczyk, R. Paulson, and K. Bendikson, "Characterizing the influence of vitamin D levels on IVF outcomes," Human Reproduction, vol. 27, no. 11, pp. 3321-3327, 2012.

[6] M. F. Holick, "High prevalence of vitamin D inadequacy and implications for health," Mayo Clinic Proceedings, vol. 81, no. 3, pp. 353-373, 2006.

[7] D. Bassil, M. Rahme, M. Hoteit, and G. E.-H. Fuleihan, "Hypovitaminosis D in the Middle East and North Africa," Dermato-Endocrinology, vol. 5, no. 2, pp. 274-298, 2013.

[8] M. Chakhtoura, M. Rahme, N. Chamoun, and G. El-Hajj Fuleihan, "Vitamin D in the Middle East and North Africa," Bone Reports, vol. 8, pp. 135-146, 2018.

[9] M. F. Holick, "Vitamin D deficiency," New England Journal of Medicine, vol. 357, no. 3, pp. 266-281, 2007.

[10] J. S. Adams and M. Hewison, "Unexpected actions of vitamin D: new perspectives on the regulation of innate and adaptive immunity," Nature Clinical Practice Endocrinology \& Metabolism, vol. 4, no. 2, pp. 80-90, 2008.

[11] P. Autier and S. Gandini, "Vitamin D supplementation and total mortality," Archives of Internal Medicine, vol. 167, no. 16, pp. 1730-1737, 2007.

[12] J. Wortsman, L. Y. Matsuoka, T. C. Chen, Z. Lu, and M. F. Holick, "Decreased bioavailability of vitamin D in obesity," The American Journal of Clinical Nutrition, vol. 72, no. 3, pp. 690-693, 2000.

[13] D. D. Bikle, "Vitamin D metabolism, mechanism of action, and clinical applications," Chemistry \& Biology, vol. 21, no. 3, pp. 319-329, 2014.

[14] T. Sakaki, N. Kagawa, K. Yamamoto, and K. Inouye, "Metabolism of vitamin D3 by cytochromes P450," Frontiers in Bioscience: A Journal and Virtual Library, vol. 10, pp. 119-134, 2005.

[15] S. Christakos, P. Dhawan, A. Verstuyf, L. Verlinden, and G. Carmeliet, "Vitamin D: metabolism, molecular mechanism of action, and pleiotropic effects," Physiol Rev, vol. 96, no. 1, pp. 365-408, 2016.

[16] J. S. Adams, B. Rafison, S. Witzel et al., "Regulation of the extrarenal CYP27B1-hydroxylase," The Journal of Steroid Biochemistry and Molecular Biology, vol. 144, pp. 22-27, 2014.

[17] M. Kamao, S. Tatematsu, S. Hatakeyama et al., "C-3 epimerization of vitamin D3 metabolites and further metabolism of C-3 epimers: 25-hydroxyvitamin D3 is metabolized to 3-epi25-hydroxyvitamin D3 and subsequently metabolized through C-1alpha or C-24 hydroxylation," The Journal of Biological Chemistry, vol. 279, no. 16, pp. 15897-15907, 2004.

[18] R. L. Schleicher, S. E. Encisco, M. Chaudhary-Webb, E. Paliakov, L. F. McCoy, and C. M. Pfeiffer, "Isotope dilution ultra performance liquid chromatography-tandem mass spectrometry method for simultaneous measurement of 25hydroxyvitamin D2, 25-hydroxyvitamin D3 and 3-epi-25hydroxyvitamin D3 in human serum," Clinica Chimica Acta, vol. 412, no. 17-18, pp. 1594-1599, 2011.

[19] F. Molnar, R. Sigueiro, Y. Sato et al., "1alpha,25(OH)2-3-epivitamin D3, a natural physiological metabolite of vitamin D3: its synthesis, biological activity and crystal structure with its receptor," PloS One, vol. 6, no. 3, Article ID e18124, 2011. 
[20] A. Nakashima, K. Yokoyama, T. Yokoo, and M. Urashima, "Role of vitamin D in diabetes mellitus and chronic kidney disease," World Journal of Diabetes, vol. 7, no. 5, pp. 89-100, 2016.

[21] L. L. Husemoen, B. H. Thuesen, M. Fenger et al., "Serum $25(\mathrm{OH}) \mathrm{D}$ and type 2 diabetes association in a general population: a prospective study," Diabetes Care, vol. 35, no. 8, pp. 1695-1700, 2012.

[22] K. C. Chiu, A. Chu, V. L. Go, and M. F. Saad, "Hypovitaminosis $\mathrm{D}$ is associated with insulin resistance and beta cell dysfunction," The American Journal of Clinical Nutrition, vol. 79, no. 5, pp. 820-825, 2004.

[23] E. Angellotti and A. G. Pittas, "The role of vitamin D in the prevention of type 2 diabetes: to D or not to D?" Endocrinology, vol. 158, no. 7, pp. 2013-2021, 2017.

[24] M. J. Berridge, "Vitamin D deficiency and diabetes," Biochemical Journal, vol. 474, no. 8, pp. 1321-1332, 2017.

[25] H. K. Osorio Landa, I. Perez Diaz, S. D. C. Laguna Barcenas et al., "Association of serum vitamin D levels with chronic disease and mortality," Nutricion hospitalaria, Article ID 32054282, 2020.

[26] K. Al-Dabhani, K. K. Tsilidis, N. Murphy et al., "Prevalence of vitamin $\mathrm{D}$ deficiency and association with metabolic syndrome in a Qatari population," Nutrition \& Diabetes, vol. 7, no. 4, p. e263, 2017.

[27] S. Bajaj, R. P. Singh, N. C. Dwivedi, K. Singh, A. Gupta, and M. Mathur, "Vitamin D levels and microvascular complications in type 2 diabetes," Indian Journal of Endocrinology and Metabolism, vol. 18, no. 4, pp. 537-541, 2014.

[28] H. Wan, Y. Wang, K. Zhang et al., "Associations between vitamin $\mathrm{D}$ and microvascular complications in middle-aged and elderly diabetic patients," Endocrine Practice, vol. 25, no. 8, pp. 809-816, 2019.

[29] J. G. Deckers, F. G. Schellevis, and D. M. Fleming, "WHO diagnostic criteria as a validation tool for the diagnosis of diabetes mellitus: a study in five European countries," The European Journal of General Practice, vol. 12, no. 3, pp. 108-113, 2006.

[30] A. Giustina, R. A. Adler, N. Binkley et al., "Controversies in vitamin D: summary statement from an international conference," The Journal of Clinical Endocrinology and Metabolism, vol. 104, no. 2, pp. 234-240, 2019.

[31] Y. Zhang, W. Xia, P. Lu, and H. Yuan, "The association between VDR gene polymorphisms and diabetic retinopathy susceptibility: a systematic review and meta-analysis," BioMed Research International, vol. 2016, Article ID 5305282, 2016.

[32] S. Chen, Y. Sun, and D. K. Agrawal, "Vitamin D deficiency and essential hypertension," Journal of the American Society of Hypertension, vol. 9, no. 11, pp. 885-901, 2015.

[33] R. Scragg, M. Sowers, C. Bell, H. Third National, and S. Nutrition Examination, "Serum 25-hydroxyvitamin D, diabetes, and ethnicity in the Third National Health and Nutrition Examination Survey," Diabetes Care, vol. 27, no. 12, pp. 2813-2818, 2004.

[34] E. Liu, J. B. Meigs, A. G. Pittas et al., "Predicted 25hydroxyvitamin $\mathrm{D}$ score and incident type 2 diabetes in the Framingham Offspring Study," The American Journal of Clinical Nutrition, vol. 91, no. 6, pp. 1627-1633, 2010.

[35] C. Gagnon, Z. X. Lu, D. J. Magliano et al., "Serum 25hydroxyvitamin $\mathrm{D}$, calcium intake, and risk of type 2 diabetes after 5 years: results from a national, population-based prospective study (the Australian Diabetes, Obesity and Lifestyle study)," Diabetes Care, vol. 34, no. 5, pp. 1133-1138, 2011.

[36] S. Lim, M. J. Kim, S. H. Choi et al., "Association of vitamin D deficiency with incidence of type 2 diabetes in high-risk Asian subjects," The American Journal of Clinical Nutrition, vol. 97, no. 3, pp. 524-530, 2013.

[37] S. Devaraj, G. Jialal, T. Cook, D. Siegel, and I. Jialal, "Low vitamin $\mathrm{D}$ levels in Northern American adults with the metabolic syndrome," Hormone and Metabolic Research, vol. 43, no. 1, pp. 72-74, 2011.

[38] R. Jorde, M. Sneve, N. Emaus, Y. Figenschau, and G. Grimnes, "Cross-sectional and longitudinal relation between serum 25hydroxyvitamin D and body mass index: the Tromso study," European Journal of Nutrition, vol. 49, no. 7, pp. 401-407, 2010.

[39] I. H. de Boer, L. F. Tinker, S. Connelly et al., "Calcium plus vitamin $\mathrm{D}$ supplementation and the risk of incident diabetes in the Women's Health Initiative," Diabetes Care, vol. 31, no. 4, pp. 701-707, 2008.

[40] E. Angellotti, D. D’Alessio, B. Dawson-Hughes et al., "Vitamin $\mathrm{D}$ supplementation in patients with type 2 diabetes: the vitamin D for established type 2 diabetes (DDM2) study," Journal of the Endocrine Society, vol. 2, no. 4, pp. 310-321, 2018. 